\title{
Silver nanoparticle incorporation into flexible polyamide 12 membranes
}

\author{
Maria Gabriela Martins de Souza ${ }^{1}$. Jéssica Potomatti Batista ${ }^{1}$ - Emerson Henrique de Faria ${ }^{1}$ Katia Jorge Ciuffi ${ }^{1}$. \\ Lucas Alonso Rocha ${ }^{1}$ - Eduardo José Nassar $\mathbb{1}^{1} \cdot$ Jorge Vicente Lopes da Silva $^{2} \cdot$ Marcelo Fernandes Oliveira $^{2}$. \\ Izaque Alves Maia ${ }^{2}$
}

Received: 30 April 2021 / Accepted: 20 November 2021 / Published online: 20 January 2022

(c) The Author(s), under exclusive licence to Springer Science+Business Media, LLC, part of Springer Nature 2022

\begin{abstract}
To meet the demands of the market and society, the development of structured polymeric materials for application in the medical field is constantly increasing. Over the last decades, metallic silver nanoparticles have been explored due to their antimicrobial action. Here, we aimed to incorporate metallic silver nanoparticles into polymeric pieces obtained by additive manufacture via a chemical route involving silver nitrate and sodium borohydride. Polyamide 12 membranes were obtained by selective laser sintering, which was followed by washing, pretreatment, and functionalization with the alkoxides tetraethylorthosilicate and 3-aminopropyl tetraethoxysilane. For nanoparticle preparation and incorporation, a chemical route was tested under different conditions. The samples were characterized by techniques, such as X-ray diffraction, ultravioletvisible spectroscopy, and infrared vibrational spectroscopy. Nanoparticle formation and incorporation into the polyamide 12 membranes were demonstrated by the absorbance band at $420 \mathrm{~nm}$, which indicated that the particles measured between 10 and $50 \mathrm{~nm}$ in size; by the X-ray diffraction peaks at $2 \theta=38,44$, and $64^{\circ}$, which are typical of crystalline silver; and by vibrational spectroscopy, which evidenced that the nanoparticles interacted with the polyamide 12 nitrogen groups. Polyamide 12 membranes containing metallic silver nanoparticles have promising biomedical applications as antimicrobial wound dressings associated with drug carriers.
\end{abstract}

\section{Graphical Abstract}

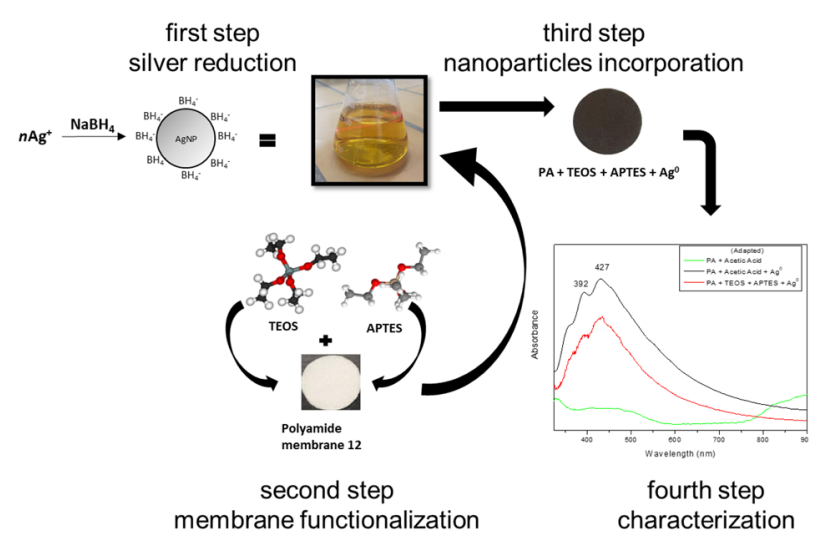

Eduardo José Nassar

eduardo.nassar@unifran.edu.br

1 Universidade de Franca, Av. Dr. Armando Salles Oliveira, 201 Pq. Universitário, Franca, SP CEP 14404-600, Brasil
Centro da Tecnologia da Informação Renato Archer (CTI) - Rod. Dom Pedro I, Km 143,6, Campinas, SP 13069-901, Brasil 
Keywords Additive manufacture $\cdot$ Biomedical applications $\cdot$ Sol-gel process $\cdot$ Functionalized polymer

\section{Highlights}

- Nanoparticle size and shape determine nanoparticle stabilization in a PA12 polymer.

- The polymer pieces obtained by additive manufacture and modified by chemical agents contribute to new applications.

- The sol-gel process is an important technique to functionalize polymeric materials.

\section{Introduction}

To meet the demands of the market and society, scientific research geared toward the development of materials and technology for application in the biomedical field has been increasing. In this scenario, the use of Additive Manufacture (AM) or 3D printing is advantageous: it requires a smaller number of fabrication steps, reduces manufacturing costs, and affords geometric freedom, contributing to the production of prostheses, ortheses, biomodels for surgical planning, controlled drug release devices, and wound dressings, among other applications [1].

By means of the Three-dimensional Technology Hub, the Renato Archer Information Technology Center, an agency linked to the Brazilian Ministry of Science, Technology, Innovations, and Communications, has stood out as a producer of pieces obtained by AM both nationwide and worldwide. Among such pieces, polyamide 12 (PA12) membranes, obtained by the Selective Laser Sintering (SLS) technique, are noteworthy. Compared to other polyamides, PA12 absorbs less water due to its relatively long hydrocarbon chain, and it displays flexibility, controlled porosity, high resistance to impact even at low temperatures, excellent stress crack resistance, high processability, good fatigue resistance under high-frequency cyclic loading, and excellent resistance to commonly employed organic solvents [2]. Therefore, PA12 membranes are good candidates for use as wound dressings and drug carriers, as already observed in other works by our group [3-6].

The appearance and spread of microorganisms that cause infectious diseases has led to the development of antibiotic resistance, which has challenged researchers in the medical field. In this context, materials consisting of metallic silver nanoparticles (AgNPs) have emerged as a new class of antimicrobial agents in the biomedical area: AgNPs have strong bactericidal effect and do not induce microbial resistance to conventional antibiotics [7]. This is due to the nanometric size of the particles, which presents distinct and sometimes superior properties as compared to particles on the micro- and/or macroscopic scale [8]. Even at low concentrations, AgNPs present high antibacterial potential against an array of microorganisms given that they have affinity for functional groups containing thiol, phosphate, hydroxyl, and amine, which are present in proteins, enzymes, DNA, and RNA [9]. In this way, AgNPs can adhere to the surface of the negatively charged cell membrane, causing protein denaturation and cell death. AgNPs can also alter the cell membrane functionality, preventing bacterial regeneration [7, 8]. Indeed, recent studies have demonstrated that nanotechnology can effectively aid the fight against microorganisms [10-15].

The introduction of composites into polymers for applications in AM has been reported. A recent review has emphasized the importance of antimicrobial polymers in the context of COVID-19 [16]. Laser ablation has been used to synthesize nanoparticles for incorporation into PA12 powder [17]. Another work has described incorporation of silver and copper nanoparticles into 3D polymer nanocomposites to enhance their mechanical strength, thermal stability, and electrical conductivity [18]. AgNP preparation by thermal reduction during extrusion has also been described [19]. All such methodologies involve nanoparticle incorporation into polymers before their use in AM. Here, we propose using two different methodologies to synthesize AgNPs and to incorporate them into PA12 membranes obtained by AM, aiming at applying the resulting materials as topical wound dressings for protection against microorganisms. To this end, we prepared PA12 membranes incorporating AgNPs by the LSL technique and characterized them by UV-Vis spectroscopy, X-ray diffractometry (XRD), vibrational spectroscopy, scanning electron microscopy (SEM), and energy dispersive X-ray (EDX) analysis.

\section{Materials and methods}

The Information Technology Center Renato Archer provided the flexible PA12 membranes obtained by SLS.

Silver nitrate $\left(\mathrm{AgNO}_{3}, 100 \%\right.$ purity), sodium borohydride $\left(\mathrm{NaBH}_{4}, 99 \%\right.$ purity), ethanol (EtOH, $99.5 \%$ purity), tetraethylorthosilicate (TEOS, $98 \%$ purity), and 3-aminopropyltriethoxysilane (APTES, 98\% purity) were purchased from Sigma-Aldrich.

Solid-state spectroscopy by diffuse reflectance was performed on an Ocean Optics device equipped with a probe, a pulsed Xe PX-2 lamp, and a QE65000 detector.

XRD was conducted on an Rigaku Geigerflex D/Max-c equipped with a $\mathrm{CuK} \alpha(\lambda=1.5405 \AA)$ radiation monochromator. The XRD patterns were recorded at $2 \theta$ values ranging from 5 to $70^{\circ}$, with steps of $0.01^{\circ} / 5 \mathrm{~s}$. 
Infrared spectroscopy was carried out on a FTIR PerkinElmer Frontier spectrometer operating between 400 and $4000 \mathrm{~cm}^{-1}$ and equipped with an ATR accessory (FTIR absorption spectroscopy by total attenuated reflectance); 16 scans were averaged.

The EDS and SEM measurements were conducted on a TESCAN microscope (model VEJA 3 SBH) equipped with a $30 \mathrm{kV}$ filament and a BSE detector; the resolution was $3 \mathrm{~nm}$. The samples were coated with a gold-palladium alloy on a sputter coater QUORUM mo. SC7620 for $120 \mathrm{~s}$.

\subsection{Membrane preparation}

PA12 membranes (measuring $5.0 \times 7.0 \mathrm{~cm}$ and with thickness of $200 \mu \mathrm{m}$ ) were prepared as described previously [5]. Then, the PA12 membranes were cut into circular shape with diameter of $1 \mathrm{~cm}$, placed in a $50 \mathrm{~mL}$ beaker containing distilled water, and subjected to constant magnetic stirring for $2 \mathrm{~h}$. Next, the PA12 membranes were dried at room temperature, and excess PA powder, originating from the raw material during SLS, was removed.

\subsection{Pretreatment of PA12 membranes}

The PA12 membrane surface was activated with acetic acid solution as described previously $[5,20]$. This step protonated the PA12 -NH groups, to enhance interaction between AgNPs and the PA12 membranes. More specifically, the circular PA12 membranes were added to $1.0 \mathrm{~mol}$ $\mathrm{L}^{-1}$ acetic acid and kept under constant stirring at room temperature for $24 \mathrm{~h}$. The PA12 membranes were removed, washed with distilled water in an ultrasonic bath for $15 \mathrm{~min}$, placed on Petri dishes, and dried at room temperature.

\subsection{PA12 membrane functionalization with TEOS and APTES}

The PA12 membranes were functionalized by the sol-gel process as described previously [5]. The pretreated PA12 membranes were placed in a two-neck round-bottom flask containing $15.0 \mathrm{~mL}$ of EtOH, $3.7 \mathrm{~mL}$ of TEOS, $3.7 \mathrm{~mL}$ of APTES, and $5.0 \mathrm{~mL}$ of distilled water. The reaction mixture was kept under constant stirring at $80^{\circ} \mathrm{C}$ for $24 \mathrm{~h}$. Then, the functionalized PA12 membranes were removed from the solution, washed with distilled water in an ultrasonic bath for $15 \mathrm{~min}$, and dried at room temperature.

\subsection{Synthesis and incorporation of metallic silver nanoparticles into PA12 membrane by the Creighton method}

AgNPs were synthesized and incorporated into the pretreated PA12 membrane (either functionalized or unfunctionalized), by the Creighton method [21]. A recently prepared $2.0 \times 10^{-2} \mathrm{~mol} \mathrm{~L}^{-1} \mathrm{NaBH}_{4}$ solution was left to stand for $15 \mathrm{~min}$ in an ice bath. Next, this solution was added to an Erlenmeyer flask containing the PA12 membrane, and placed on a magnetic stirring plate. The mixture was stirred at $250 \mathrm{rpm}$; the system was kept in an ice bath. With the aid of a dropper, $25 \mathrm{~mL}$ of a $1.0 \times 10^{-2} \mathrm{~mol} \mathrm{~L}^{-1}$ $\mathrm{AgNO}_{3}$ solution was added over the $\mathrm{NaBH}_{4}$ solution at a rate of one drop per second. During addition, the mixture acquired the strong yellow color typical of metallic silver. The PA12 membrane incorporating AgNPs was kept under constant stirring in an ice bath for $30 \mathrm{~min}$. After this time, the resulting membranes were washed with distilled water in an ultrasonic bath for $15 \mathrm{~min}$ and dried at room temperature.

\subsection{Synthesis and incorporation of silver nanoparticles into PA12 membrane by the adapted methodology}

A $1.0 \times 10^{-2} \mathrm{~mol} \mathrm{~L}^{-1} \mathrm{AgNO}_{3}$ solution was added to an Erlenmeyer flask wrapped in aluminum foil and containing the pretreated PA12 membrane (either functionalized or unfunctionalized). The system was heated to $60{ }^{\circ} \mathrm{C} ; 10 \mathrm{~mL}$ of a $2.0 \times 10^{-2} \mathrm{~mol} \mathrm{~L}^{-1} \mathrm{NaBH}_{4}$ solution was added to the Erlenmeyer dropwise, at a rate of one drop per second. The mixture was kept under constant stirring at $60{ }^{\circ} \mathrm{C}$ for $24 \mathrm{~h}$. After this time, the PA12 membrane incorporating AgNPs was removed and dried at room temperature. This adapted AgNP synthesis aimed to enhance AgNP incorporation into the PA12 membranes via contact between the $\mathrm{AgNO}_{3}$ solution and the PA12 membrane upon increased temperature, to promote larger spacing between the PA12 chains. All the PA12 membranes were washed with distilled water in an ultrasonic bath for $15 \mathrm{~min}$ and dried at room temperature.

\section{Results and discussion}

A typical feature of nanoparticles is their strong coloration, caused by absorption of electromagnetic radiation in resonance with surface plasmons. Figure $1 \mathrm{~A}-\mathrm{C}$ show photographs of a colloidal AgNP solution obtained by the Creighton method (Fig. 1A) and of the pretreated PA12 membrane obtained after AgNP synthesis and incorporation by the Creighton (Fig. 1B) or the adapted (Fig. 1C) method.

AgNP color, size, and shape are directly related to visual perception. Figure 2 displays the UV-Vis absorption spectrum of the colloidal AgNP solution shown in Fig. 1A. Optical excitation induced plasmon resonance in the metal nanostructures. Figure 2 presents the surface resonance plasmon effect. This phenomenon occurs when interaction 
Fig. 1 Photographs of colloidal AgNP solution (A) and pretreated PA12 membrane after AgNP synthesis and incorporation by the Creighton (B) or the adapted method (C)

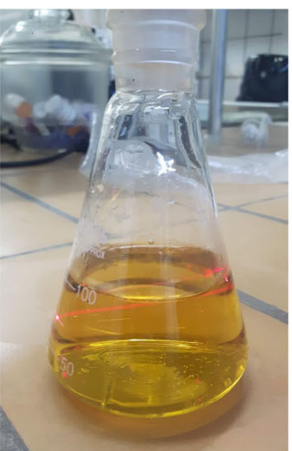

A

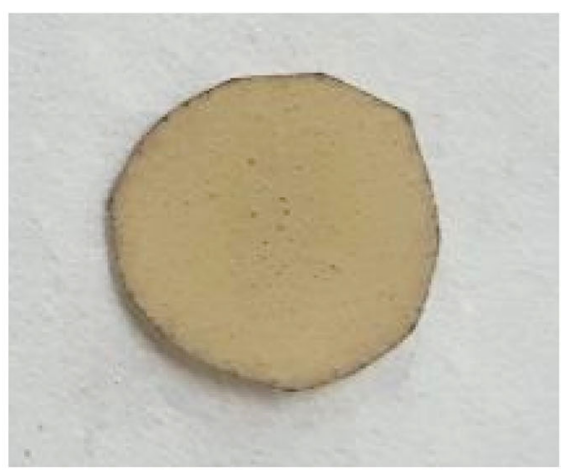

B

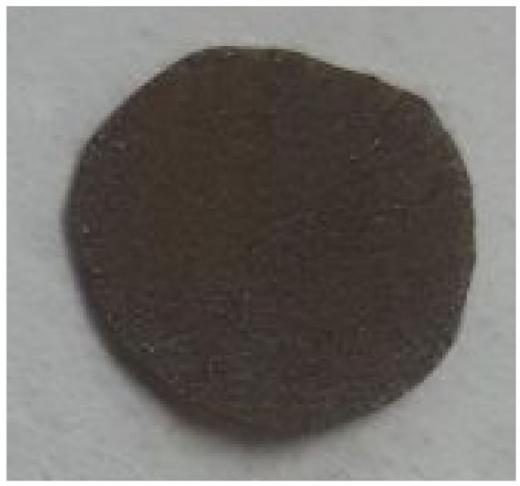

C

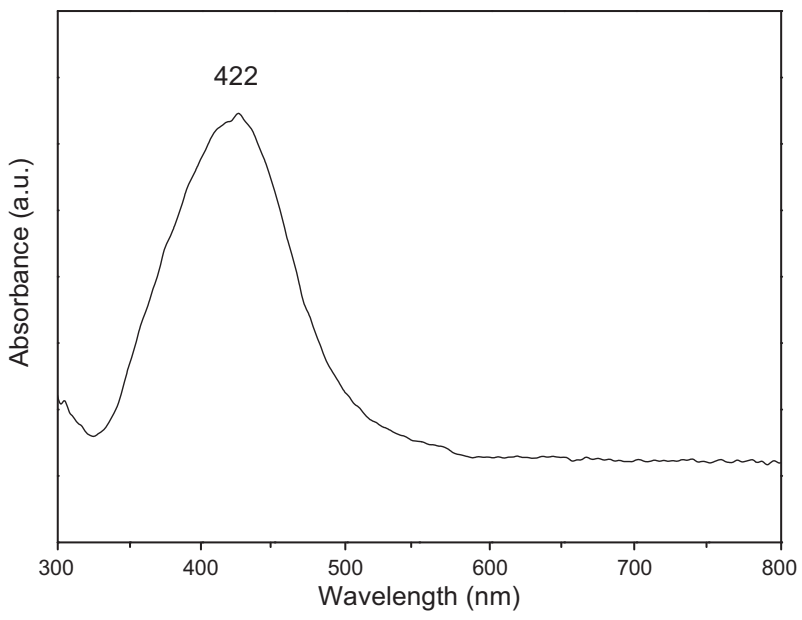

Fig. 2 UV-Vis absorbance spectrum of colloidal AgNPs

with an electromagnetic field induces collective electron oscillation, generating the plasmonic surfaces, represented in the form of a band in the absorbance spectrum [22, 23].

According to Solomon et al. (2007) [18], nanoparticle size, shape, and dispersion are directly related to their UV-Vis spectrum, as depicted in Table 1. Based on these data, the AgNPs synthesized herein measured between 35 and $50 \mathrm{~nm}$.
Table 1 Correlation between particle size (nm) and maximum absorbance wavelength (nm)

\begin{tabular}{ll}
\hline Particle size $(\mathrm{nm})$ & Maximum absorption wavelength $(\mathrm{nm})$ \\
\hline $5-10$ & $380-390$ \\
$10-14$ & $395-405$ \\
$35-50$ & $420-435$ \\
$60-80$ & $438-450$
\end{tabular}

Source: Solomon et al. (2007)

Figure $3 \mathrm{~A}$ and $\mathrm{B}$ present the $\mathrm{UV}-\mathrm{Vis}$ absorbance spectra of the pretreated PA12 membranes (both unfunctionalized and functionalized) before and after AgNP synthesis and incorporation by the Creighton or the adapted method, respectively.

The spectra of the pretreated PA12 membranes (both unfunctionalized and functionalized) after AgNP synthesis and incorporation by the Creighton method (Fig. 3A) displayed bands in the region of $400 \mathrm{~nm}$, typical of AgNP plasmon resonance. The band profile comprised two bands with maxima at 391 and $420 \mathrm{~nm}$, indicating that AgNPs incorporated into the PA12 membrane had different sizes, as shown in Table 1. The absence of the bands due to AgNPs in the spectrum of the pretreated PA12 membrane is 
(A)

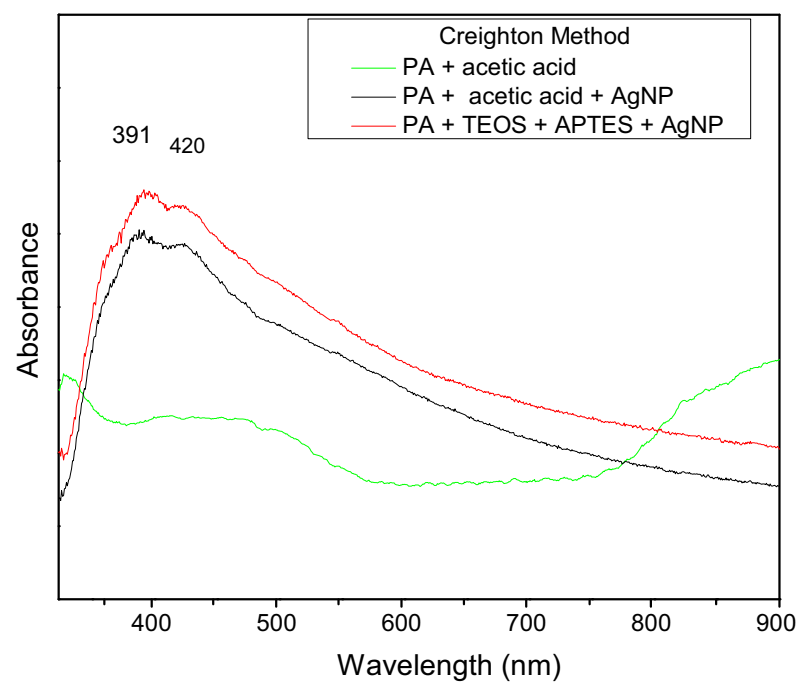

(B)

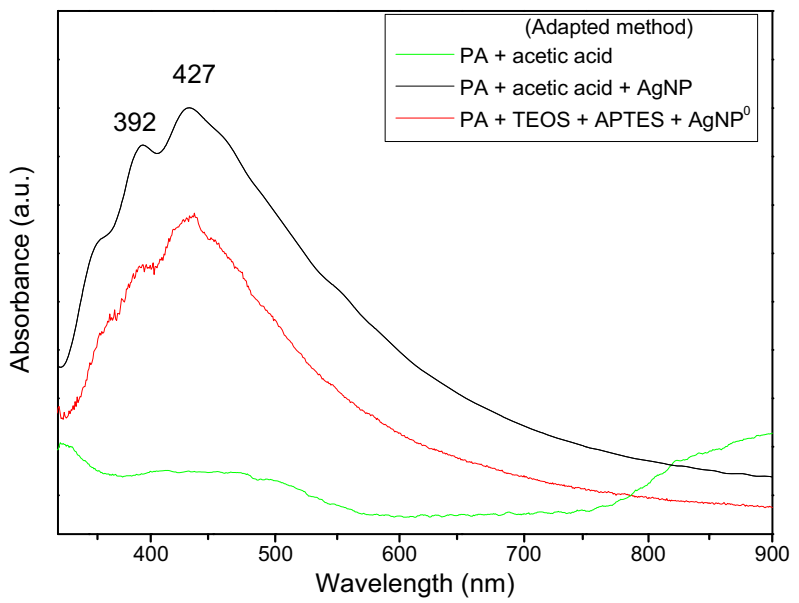

Fig. $3 \mathrm{UV}-$ Vis absorbance spectra of pretreated PA12 membrane (both unfunctionalized and functionalized) before and after AgNP synthesis and incorporation by the Creighton (A) or the adapted (B) method

consistent with AgNP formation and incorporation into the membrane. Similarly, the spectra of the pretreated PA12 membrane (both unfunctionalized and functionalized), after AgNP synthesis and incorporation via the adapted method, displayed bands at 392 and $427 \mathrm{~nm}$ (Fig. 3B), indicating that these AgNPs had approximately the same size as AgNPs prepared and incorporated by the Creighton method. However, when we observed the intensity of these bands more closely, we noted an inversion: for AgNPs obtained and incorporated by the adapted method, the band at $497 \mathrm{~nm}$ was more intense than the band at $392 \mathrm{~nm}$, which we attributed to the larger size distribution of these AgNPs as compared to AgNPs prepared and incorporated by the Creighton method. The latter method provided smaller particles $(391 \mathrm{~nm} / \sim 10 \mathrm{~nm})$ than the adapted method
$(427 \mathrm{~nm} / \sim 40 \mathrm{~nm})$. Moreover, AgNPs are known to be thermodynamically unstable and to aggregate, to minimize the total or the interface area of the system. This knowledge was reinforced by the additional heating steps introduced by the adapted methodology, which increased the aggregation rate and, hence, the particle size.

The number of plasmon resonance bands is important: according to the Mie theory [24], spherical nanoparticles are expected to have only one plasmon band, whereas particles in the shape of rods, discs, and triangles, among others, may present new bands in the UV-Vis spectrum. Thus, an absorbance band composed of various other bands indicates the lower symmetry of the particles [25].

The bandwidth may be related to the degree of particle dispersion and aggregation $[4,26]$, which can be measured by the full width at half maximum (FWHM). The wider the band, the more aggregated the particles. Table 2 lists the FWHM for AgNPs in suspension and after their preparation and incorporation into the pretreated PA12 membrane (both unfunctionalized and functionalized).

On the basis of the data in Table 2, it is concluded that the AgNPs incorporated into the pretreated PA12 membrane (both unfunctionalized and functionalized) were more aggregated than those in solution. A comparison between the two methodologies for AgNP synthesis and incorporation showed that the Creighton method led to greater aggregation than the adapted method; the FWHM values were 213.9 and $148.0 \mathrm{~nm}$ for the pretreated, nonfunctionalized PA12 membranes, respectively. For both methods, functionalization of the PA12 membrane resulted in lower FWHMs, which is attributed to stabilization of the AgNPs against aggregation due to interactions with the APTES amine groups $\left(-\mathrm{NH}_{2}\right)$.

The pretreated PA12 membranes were characterized by XRD before and after functionalization with TEOS and APTES, and before and after AgNP incorporation. Figure 4 shows the XRD patterns of the pretreated PA12 membrane before and after functionalization. According to Campos et al. [20], pretreated membranes present a semicrystalline region due to the presence of hydrogen bonds between their chains, forming the $\gamma$ phase with diffraction peak at $2 \theta=$ $21.16^{\circ}$. Upon functionalization with alkoxide, the intensity of the diffraction peak decreases, which is presumably related to membrane coating.

According to Liu et al. [27], thermal expansion of the hydrogen bond may be triggered by heat-treatment of the PA12 membrane at $60^{\circ} \mathrm{C}$, causing the XRD peak to shift toward smaller $2 \theta$ values. Here, the peak shifted to $21.89^{\circ}$ after functionalization, indicating that the added alkoxides may have inserted between the polymeric chains of the pretreated PA12 membrane, disrupting the hydrogen bonds. The $2 \theta$ values of the pretreated, non-functionalized PA12 membrane and of the pretreated, functionalized PA 
Table 2 FWHM of the absorbance bands of AgNPs in suspension and prepared and incorporated into the pretreated PA12 membrane (both unfunctionalized and functionalized) by the Creighton or the adapted method

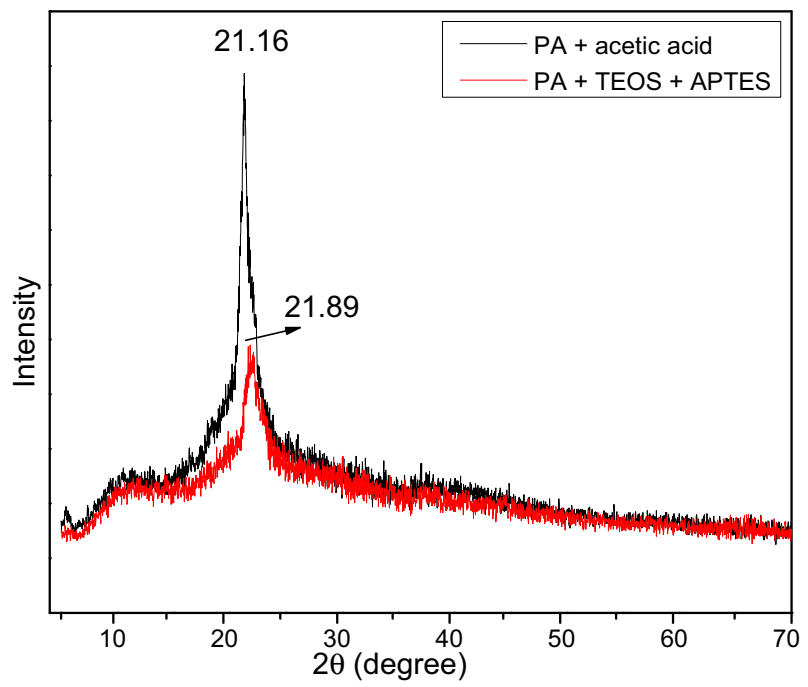

Fig. 4 XRD patterns of pretreated PA12 membranes before and after functionalization

membrane at $21.16^{\circ}$ and $21.89^{\circ}$, respectively corresponded to d spacings of 2.12 and $2.07 \AA$, respectively, proving that the interlayer spacing did not change significantly within the pretreated, functionalized PA12 membrane.

The XRD patterns of the PA12 membranes after incorporation of AgNPs are shown in Fig. 5A, B for samples produced by the Creighton and Adapted methods, respectively.

In Fig. 5A, the XRD patterns of the samples prepared via the Creighton method did not display any peaks associated with the AgNPs, probably because only a small amount of AgNPs was incorporated, falling below the detection limit of the equipment. Figure 1B had revealed a slightly colored membrane, indicating that AgNPs were present, as confirmed by the UV-Vis spectra in Fig. 3A. In Fig. 5B, the XRD patterns of the samples prepared via the Adapted method displayed peaks at $2 \theta=37.8,44.1$, and $64.6^{\circ}$, which we respectively attributed to the characteristic (111), (200), and (220) diffraction planes of metallic silver with face centered cubic structure [8]. The (111) plane is one of the most important: according to the literature, this plane contains the largest amount of exposed silver atoms, which leads to higher reactivity, positively affecting the bactericidal action of the material [28].

The FTIR spectrum of the pretreated PA12 membrane, Fig. 6, displayed the bands at 1639 and $3294 \mathrm{~cm}^{-1}$,

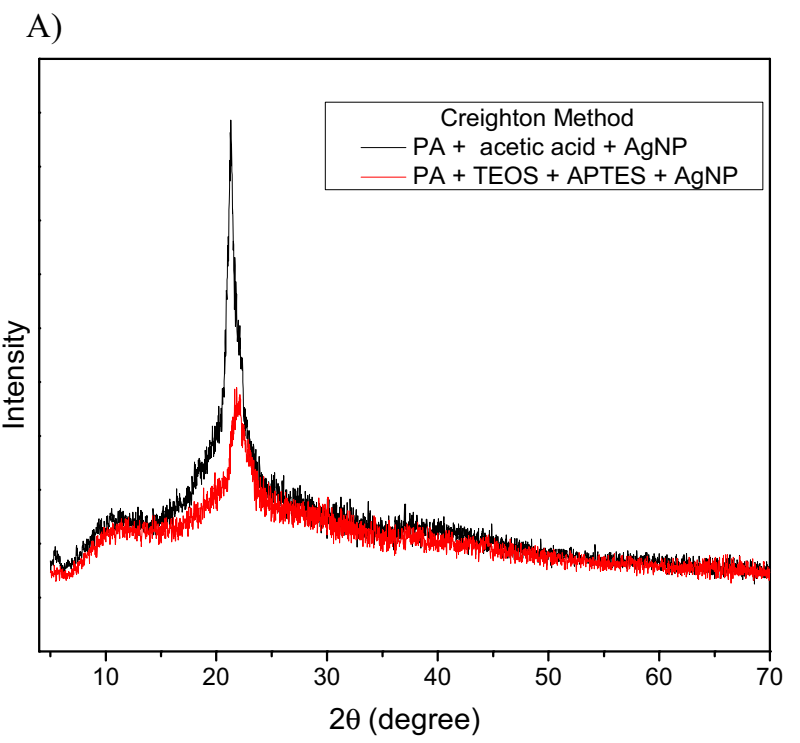

B)

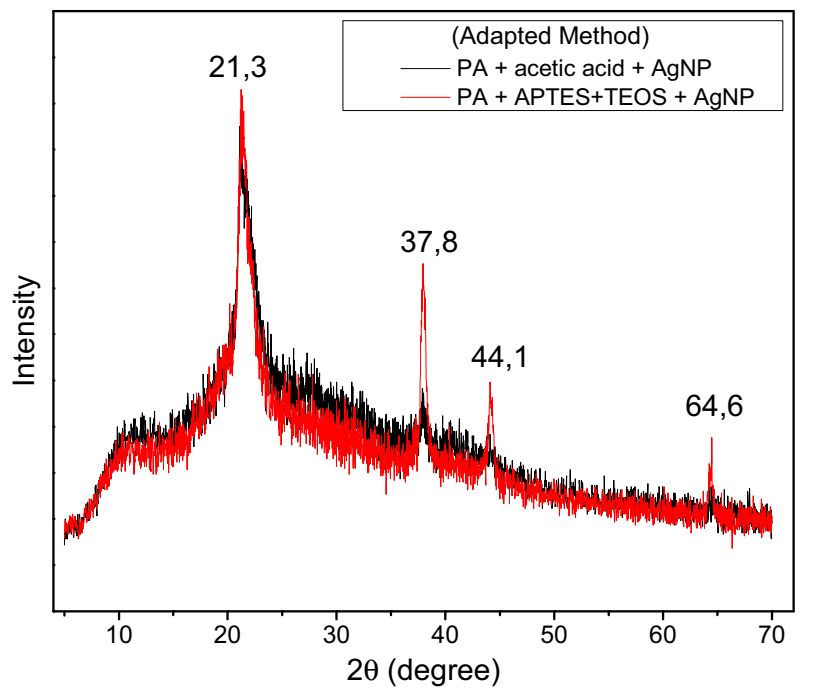

Fig. 5 XRD patterns of flexible PA12 membranes incorporated with AgNPs obtained by the Creighton (A) or the adapted (B) methods

corresponding to bending and stretching vibrations of the $-\mathrm{NH}_{2}$ group, respectively. The band at $1550 \mathrm{~cm}^{-1}$ was due to $-\mathrm{NH}$ deformation and $-\mathrm{CN}$ stretching of the secondary amide. The bands at 2846 and $2924 \mathrm{~cm}^{-1}$ are attributed to $\mathrm{C}-\mathrm{H}$ stretching vibrations, and the bands at 721,1153 , and $1463 \mathrm{~cm}^{-1}$ were respectively attributed to vibrational modes of $-\mathrm{NH}$ deformation and to the $\mathrm{C}-\mathrm{CO}-\mathrm{NH}_{2}$ and $\mathrm{C}=\mathrm{O}$ groups of the primary amide. The bands in the range between 1157 


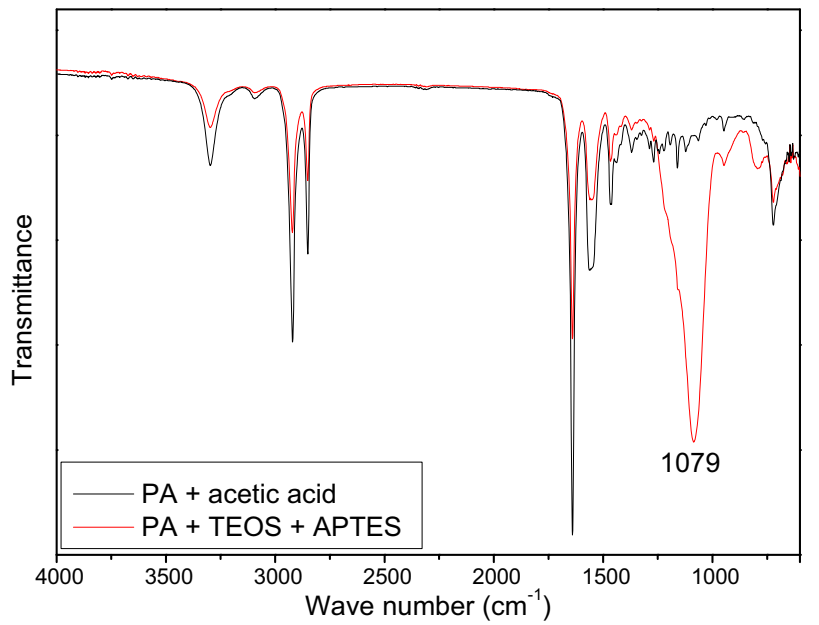

Fig. 6 FTIR of pretreated PA12 membrane before and after functionalization

and $949 \mathrm{~cm}^{-1}$ were related to amorphous and crystalline PA12 structures; the band at $1157 \mathrm{~cm}^{-1}$ was assigned to the amide and methylene groups and/or to combinations of $-\mathrm{NH}$ deformation and $\mathrm{O}=\mathrm{C}-\mathrm{N}$ stretching. The band at $949 \mathrm{~cm}^{-1}$ was ascribed to $-\mathrm{NH}$ deformation $[5,20]$.

The spectrum of the pretreated, functionalized PA12 membrane presented an intense band at $1078 \mathrm{~cm}^{-1}$, typical of the Si-O-Si group, and at $943 \mathrm{~cm}^{-1}$, due to the $\mathrm{Si}-\mathrm{OH}$ bond, confirming that the PA12 membrane was functionalized using TEOS and APTES, as reported by Souza et al. [3]. and Kim et al. [29].

Figure 7 illustrates the spectra of the pretreated PA12 membranes following incorporation of AgNPs by the Creighton or the Adapted method. The spectrum of the pretreated, functionalized PA12 membrane presented bands around $1079 \mathrm{~cm}^{-1}$, typical of $\mathrm{Si}-\mathrm{O}-\mathrm{Si}$ vibrations arising from hydrolysis and condensation of TEOS and APTES. The spectrum of the pretreated, functionalized PA12 membranes after incorporation of AgNPs by the Creighton or the Adapted method displayed a band at 1412 and $1435 \mathrm{~cm}^{-1}$, respectively. The intensification and shift of this band indicated interaction between the $-\mathrm{CN}$ and $-\mathrm{NH}$ nitrogen groups in PA12. In addition, the band at $1079 \mathrm{~cm}^{-1}$, attributed to the Si-O-Si group, shifted to lower wavenumber, indicating that heavier atoms interacted with oxygen atoms of the silica network. AgNPs have high affinity for compounds bearing oxygen and nitrogen atoms; e.g., APTES presents the terminal $-\mathrm{NH}_{2}$ group, explaining the alterations in the vibrational spectra of the pretreated, functionalized PA12 membrane with incorporated AgNPs.

Figure 8A-C show SEM micrographs (backscattering mode) at different magnifications of the pretreated, functionalized PA12 membrane before and after AgNP incorporation by the Creighton or the Adapted method. The pretreated PA12 membrane (not shown) comprised grains

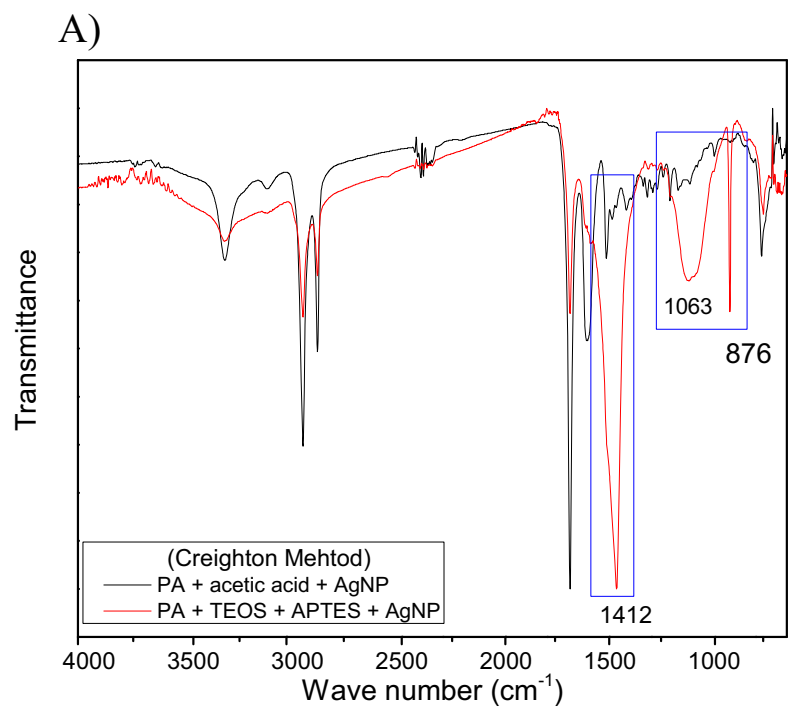

B)

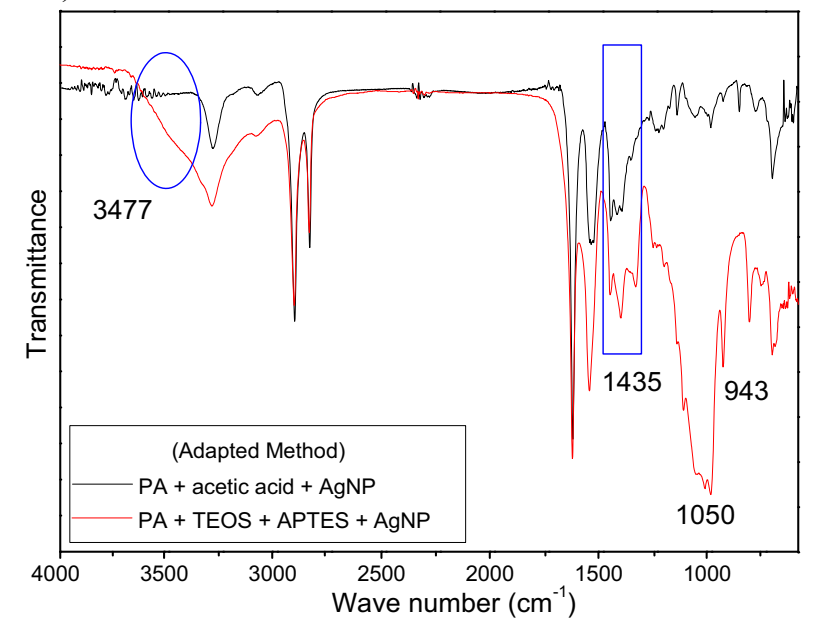

Fig. 7 FTIR spectra of pretreated PA12 membranes following incorporation of AgNPs by the Creighton (A) or the Adapted method (B)

of different sizes (ranging from $\mathrm{xxx}$ to yyy $\mu \mathrm{m}$ ), as previously described in the literature [3].

Figure 8A shows the pretreated PA12 membrane functionalized with TEOS and APTES, which exhibited a relatively homogeneous surface. In contrast, Fig. 8B revealed the presence of agglomerated AgNPs in the pretreated, functionalized PA12 membrane after AgNP incorporation by the Creighton method, which appear as white domains at higher magnification. The presence of larger quantities of AgNPs is evident in the case of samples prepared by the Adapted method (Fig. 8C), consistent with the differences in the colors of the respectively membranes illustrated in Fig. 1.

Figure 9 shows the EDX spectra of the pretreated, functionalized PA12 membranes before and after AgNP incorporation by the Creighton or the Adapted method.

The spectrum of the pretreated, functionalized PA12 membrane after AgNP incorporation by the Adapted 
Fig. 8 SEM micrographs of (A) pretreated, functionalized PA12 membrane; (B) pretreated, functionalized PA12 membrane after AgNP incorporation by the Creighton method; and (C) pretreated, functionalized PA12 membrane after AgNP incorporation by the Adapted method

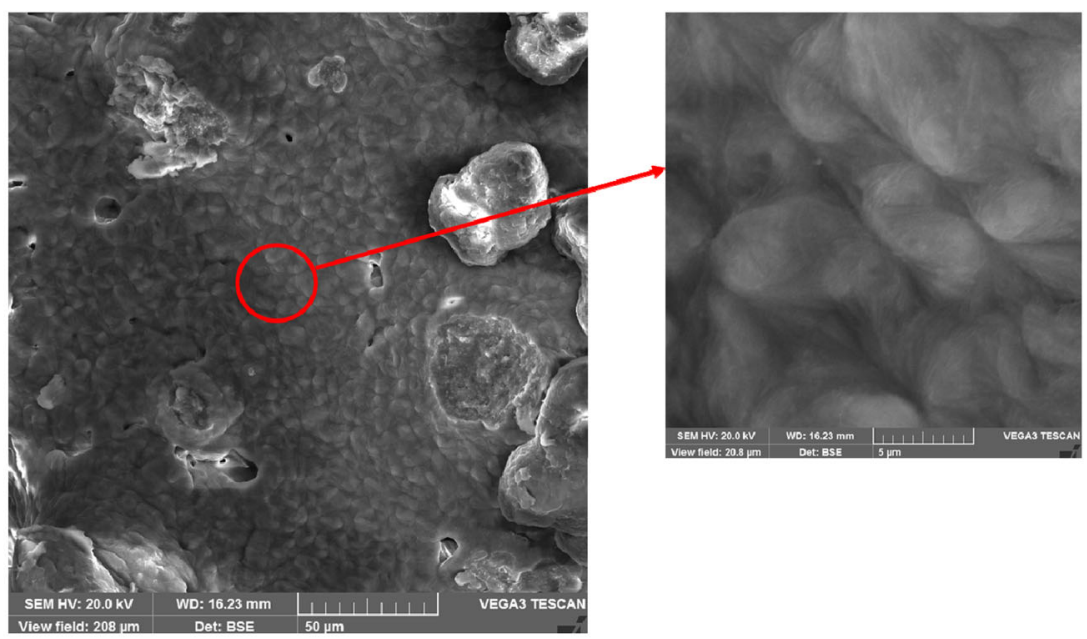

A)

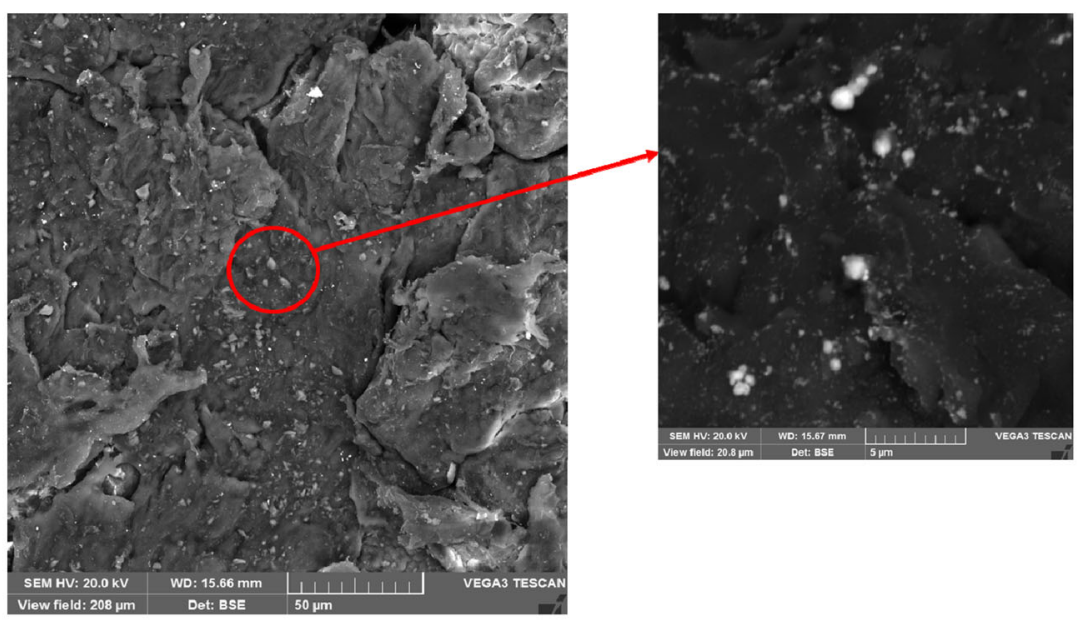

B)

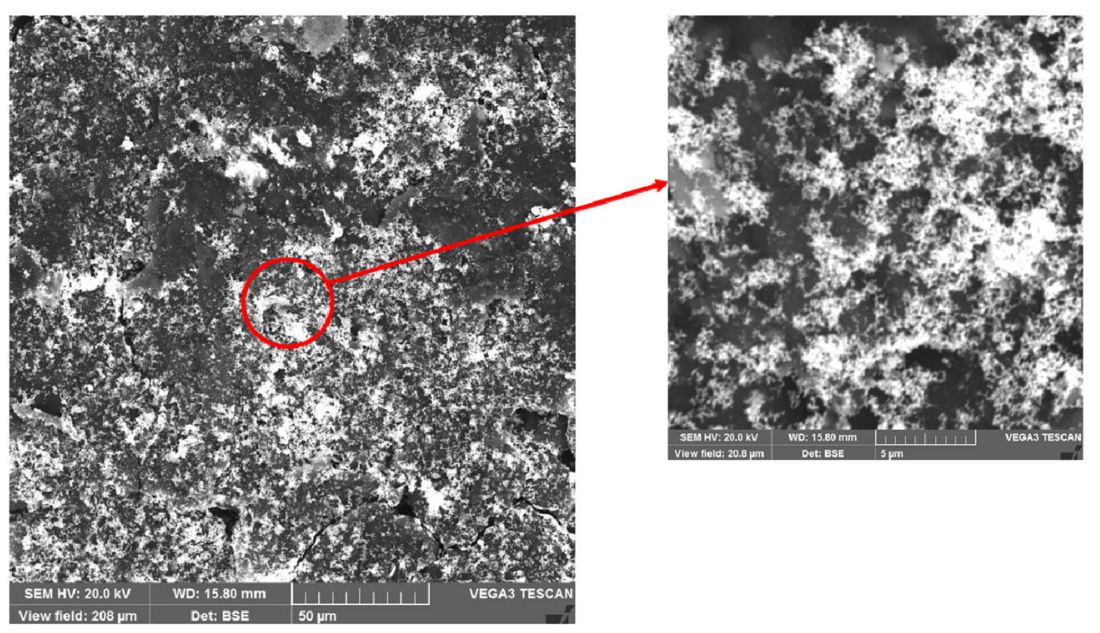

C)

method displayed a more intense peak due to AgNPs, once again confirming that the adapted method promoted incorporation of a larger amount of AgNPs into the pretreated, functionalized PA12 membrane as compared to the Creighton method, which in turn led to lower AgNP concentration in the membrane. As expected, the spectra of the 


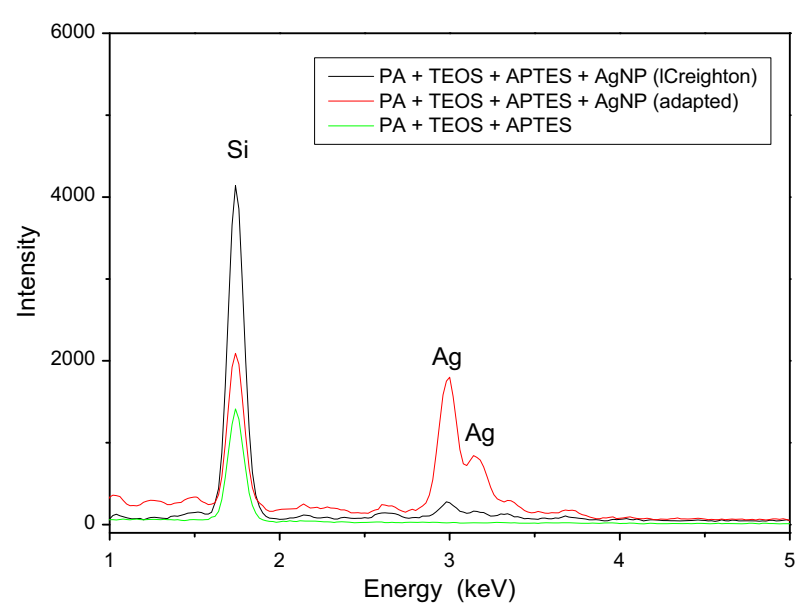

Fig. 9 EDS spectra of the pretreated, functionalized PA12 membrane before and after AgNP incorporation by the Creighton or the Adapted method

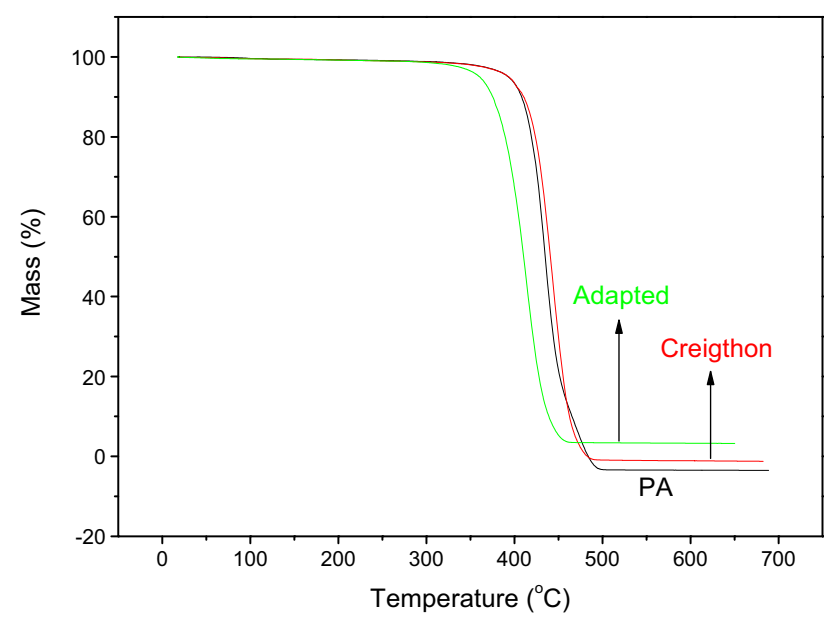

Fig. 10 TGA curves for PA12 membranes incorporated with AgNPs prepared by the Creighton method or the Adapted method

three samples all exhibited a peak due to Si, confirming functionalization of the pretreated PA12 membrane by the sol-gel methodology.

Thermogravimetric analysis enabled the amount of AgNPs incorporated into the membranes to be quantified. Figure 10 illustrates the TGA curves recorded for PA12 membranes incorporating AgNPs prepared by the Creighton method or the Adapted method. In the latter case, AgNP incorporation was $3.1 \%$, compared to $1.3 \%$ in the former case, consistent with the different membrane colors evident in Fig. 1.

\section{Conclusion}

The sol-gel methodology was shown to be suitable for functionalization of the polyamide membrane obtained by AM via the SLS technique. Functionalization was essential to modify the surface of the membrane, preparing it for the intended applications.

The metallic silver nanoparticles were incorporated into the functionalized membrane via interaction with the $-\mathrm{NH}_{2}$ of the alkoxides. The Adapted method proved efficient for incorporation of the nanoparticles. Silver nanoparticles chemically interacted with the PA12 membrane, as revealed by the shift in key diagnostic peaks in the respective infrared spectra. For the functionalized PA12 membrane, the peak ascribed to the $\mathrm{Si}-\mathrm{O}-\mathrm{Si}$ vibration mode appeared at $1079 \mathrm{~cm}^{-1}$. After silver nanoparticle incorporation, this peak was displaced to 1063 and $1050 \mathrm{~cm}^{-1}$ for nanoparticles prepared by the Creighton method and the Adapted method, respectively. These peaks indicated interaction between the nanoparticles and the PA12 membrane.

The SLS technique provided a flexible membrane, opening perspectives for biomedical applications such as wound dressings containing bactericidal agents and drugs, as mentioned in previous works by our group. The presence of alkoxides with appropriate functional groups in the membrane favors interaction with target biomolecules.

Acknowledgements This study was partially funded by Coordenação de Aperfeiçoamento de Pessoal de Nível Superior (CAPES) - Brazil Finance Code 001; Fundação de Amparo à Pesquisa do Estado de São (FAPESP, processes 2015/20298-0 (LAR), 2019/02641-0 and 2019/ 26439-5 (EJN), and 2019/20214-1 (MGMS)); and Conselho Nacional de Desenvolvimento Científico e Tecnológico (CNPq, processes 302702/2018-0 (LAR), 302668/2017-9 (EJN), and 148691/2018-8 and 123739/2019-5 (MGMS)).

\section{Compliance with ethical standards}

Conflict of interest The authors declare no competing interests.

Publisher's note Springer Nature remains neutral with regard to jurisdictional claims in published maps and institutional affiliations.

\section{References}

1. Zaparolli, D (2019) O avanço da impressão 3D. Pesquisa FAPESP. Seção Engenharia. Disponível em: https://revista pesquisa.fapesp.br/2019/02/11/o-avanco-da-impressao-3d/. Acesso em 04 Abr. 2020.

2. Touris A, Turcios A, Mintz E, Pulugurtha SR, Thor P, Jolly M, Jalgaonkar U (2020) Effect of molecular weight and hydration on the tensile properties of polyamide 12. Results Mater 8:100149-100156

3. Souza ML, Moscardini SB, de Faria EH, Ciuffi KJ, Rocha LA, Nassar EJ, Silva JVL, Oliveira MF, Maia IA (2018) Óxido de ítrio e alumínio dopado com $\mathrm{Yb} 3+$ e Er3 + incorporado em membrana de poliamida. Quím Nova 41:519-527

4. de Souza ÉA, Rocha LA, de Faria EH, Ciuffi KJ, Nassar EJ, Silva JVL, Oliveira MF, Maia IA (2018) Incorporation of the Chemotherapy Medication Cisplatin into Polyamide Membrane. J Inorg Biochem 180:171-178

5. de Souza ÉA, de Campos BM, Rocha LA, de Faria EH, Ciuffi KJ, Nassar EJ, Silva JVL, Oliveira MF, Maia IA (2016) Modificação 
de Membrana de Poliamida via Sol-Gel e Incorporação de Composto de Európio (III) Luminescente. Quím Nova 39:1044-1050

6. de Sousa KZR, de Faria EH, Marçal L, Ciuffi KJ, Ricci EG, Rocha LA, Nassar EJ, Silva JVL, Oliveira MF, Maia IA (2021) Incorporação de rodamina $\mathrm{B}$ em membrana de poliamida pelo método da adsorção. Quím Nova 44:154-160

7. Shankar PD, Shobana S, Karuppusamy I, Pugazhendhi A, Ramkumar VS, Arvindnarayan S, Kumar G (2016) A review on the biosynthesis of metallic nanoparticles (gold and silver) using biocomponents of microalgae: formation mechanism and applications. Enzym Microb Technol 95:28-44

8. Kalantari K, Mostafavi E, Afifi AM, Izadiyan Z, Jahangirian H, Rafiee-Moghaddam R, Webster TJ (2020) Wound Dressings Functionalized with Silver Nanoparticles: Promises and Pitfalls. Nanoscale 12:2268-2291

9. Marambio-Jones C, Hoek EMV (2010) A review of the antibacterial effects of silver nanomaterials and potential implications for human health and the environment. J Nanopart Res 12:1531-1551

10. Vazquez-Munoz R, Lopez-Ribot JL (2020) Nanotechnology as an Alternative to Reduce the Spread of COVID-19. Challenges $11: 15-24$

11. Jeremiah SS, Miyakawa K, Morita T, Yamaoka Y, Ryo A (2020) Potent antiviral effect of silver nanoparticles on SARS-CoV-2 Biochem. Biophys Res Comm 533:195-200

12. Raghav S, Yaday PK, Kumar D, (2020) Nanotechnology for a sustainable future Handbook of Nanomaterials for Manufacturing Applications, CHAPTER 19 https://doi.org/10.1016/B978-0-12821381-0.00019-3, Elsevier Inc. 465-492.

13. Lara HH, Ixtepan-Turrent L, Yacaman MJ, Lopez-Ribot J (2020) Inhibition of Candida auris Biofilm Formation on Medical and Environmental Surfaces by Silver Nanoparticles. ACS Appl Mater Interfaces 12:21183-21191

14. Wong JKH, Tan HK, Lau SY, Yap PS, Danquah MK (2019) Potential and challenges of enzyme incorporated nanotechnology in dye wastewater treatment: a review. J Environ Chem Eng 7:103261-103278

15. Silva MMP, Aguiar MIF, Rodrigues AB, Miranda MDC, Araújo MAM, Rolim ILTP (2017) The use of nanoparticles in wound treatment: a systematic review. Rev Esc Enferm USP 51:03272-03282

16. Yaragatti N, Patnaik A (2021) A review on additive manufacturing of polymers composites. Mater Today: Proc 44:4150-4157

17. Hupfeld T, Sommereyns A, Schuffenhauerc T, Zhuravlev E, Gann MKS, Keßler O, Schmidt M, Gökce B, Barcikowski S (2020) How colloidal surface additivation of polyamide 12 powders with welldispersed silver nanoparticles influences the crystallization already at low $0.01 \mathrm{vol} \%$. Addit Manuf 36:101419

18. Aktitiz I, Varol R, Akkurt N, Saraç MF (2020) In-situ synthesis of $3 \mathrm{D}$ printable mono- and $\mathrm{Bi}$-metallic $(\mathrm{Cu} / \mathrm{Ag})$ nanoparticles embedded polymeric structures with enhanced electromechanical properties. Polym Test 90:106724

19. Parida D, Simonetti P, Frison R, Bulbul E, Altenried S, Arroyo Y, Balogh-Michels Z, Caseri W, Ren Q, Hufenus R, Gaan S (2020) Polymer-assisted in-situ thermal reduction of silver precursors: a solventless route for silver nanoparticles-polymer composites. Chem Eng J 389:123983

20. de Campos BM, Calefi PS, Ciuffi KJ, de Faria EH, Rocha LA, Nassar EJ, Silva JVL, Oliveira MF, Maia IA (2014) Coating of polyamide 12 by sol-gel methodology. J Therm Anal Calor 115:1029-1035

21. Melo Jr MA, Santos LSS, Gonçalves MC, Nogueira AF (2012) Preparação de nanopartículas de prata e ouro: um método simples para a introdução da nanociência em laboratório de ensino. Quím Nova 35:1872-1878

22. Sheldon MT, van de Groep J, Brown AM, Polman A, Atwater HA (2014) Plasmoelectric potentials in metal nanostructures. Science 346:828

23. Solomon S, Bahadory M, Jeyarajasingam AV, Rutkowsky SA, Boritz C (2007) Synthesis and study of silver nanoparticles. J Chem Educ 84:322-325

24. Mie G (1908) Contributions to the optics of turbid media, especially colloidal metal solutions. Translated Ann der Phys 25:377-445

25. Pal S, Tak YK, Song JM (2007) Does the Antibacterial Activity of Silver Nanoparticles Depend on the Shape of the Nanoparticle? A Study of the Gram-Negative Bacterium Escherichia coli. Applied and environmental microbiology. Appl Enviromen Microb 73:1712-1720

26. Li Y, Leung P, Yao L, Song QW, Newton E (2006) Antimicrobial effect of surgical masks coated with nanoparticles. J Hospital Infect 62:58-63

27. Liu T, Chen D, Phang IY, Wei C (2014) Studies on crystal transition of polyamide 11 nanocomposites by variabletemperature X-ray diffraction Chin. J Poly Sci 32:115-122

28. Kharaghani D, Khan MQ, Shahzad A, Inoue Y, Yamamoto T, Rozet S, Kim IS (2018) Preparation and in-vitro assessment of hierarchal organized antibacterial breath mask based on polyacrylonitrile/silver (PAN/AgNPs) nanofiber. Nanomaterials 8:461-473

29. Kim YJ, Park HC, Kim BK (2015) Triple shape-memory effect by silanized polyurethane/silane-functionalized graphene oxide nanocomposites bilayer. High Perform Poly 27:886-897 